\title{
INFERRING BEHAVIOUR OF GRAZING LIVESTOCK: OPPORTUNITIES FROM GPS TELEMETRY AND ACTIVITY SENSORS APPLIED TO ANIMAL HUSBANDRY
}

\author{
Valentina Becciolini, Maria Paola Ponzetta \\ University of Florence, Italy \\ valentina.becciolini@unifi.it, mariapaola.ponzetta@unifi.it
}

\begin{abstract}
GPS technologies represent a valuable tool to study animal behaviour remotely. The research is aimed at evaluating the use of GPS collars equipped with activity sensors to infer behaviour in grazing cattle. Six cows from two breeds (Limousin, Chianina) were equipped for 8 months with GPS collars with built-in tri-axial accelerometers providing activity measurements every 152 seconds. Four behaviours (resting, rumination, feeding, walking) were directly observed in synchrony with collar measurements. Behaviours were classified with discriminant analysis (DA), considering a set of six predictors and their logarithm: movement rate (metres/hour), activity measurements on the X (forward/backward) and Y (sideways) axes and their difference, mean and variance. We tested several models and reported the ones with the highest rate of correct classifications. These were achieved by adopting separate models for the two breeds and, within breed, for two season-related periods (spring/summer and autumn). This suggests that the environmental effects (e.g., weather), as well as the breed-specific habits, have to be taken into account when inferring behaviour in grazing animals, since they produce significant alterations in intensity of activity. Walking activity was misclassified in more than $80 \%$ of cases, while rumination and resting behaviour were mutually mistaken. Feeding and walking were thus merged to obtain active behaviours, and rumination and resting were classified as inactive behaviours. On average, DA correctly classified over $90 \%$ of active intervals and $85 \%$ of inactive behaviours. In conclusion, the simultaneous use of GPS and activity sensors represents a useful technique to track movements of grazing livestock and, at the same time, to discriminate between active and inactive behaviours. This information could provide benefits for rangeland management in terms of improving their efficiency of utilisation and enhancing the productive performances of animals.
\end{abstract}

Keywords: accelerometer, GPS/GSM collars, activity budget, classification, Gaussian Finite Mixture Models.

\section{Introduction}

The study of behaviour in grazing livestock assumes an essential role in research topics related to rangeland ecology and management, as well as in animal husbandry practices. The assessment of sustainable grazing systems, aiming at the mitigation of negative impacts deriving from excessive or reduced grazing, such as biodiversity loss [1], requires specific knowledge of plant ecophysiology associated with herbivore grazing behaviour and dynamics [2]. Thus, understanding the drivers of resource selection and mechanisms adopted by animals in order to cope with environmental conditions is crucial in free-ranging systems [3]. In livestock intensive farming, the continuous monitoring of behaviour provides farmers the opportunity to infer health conditions and well-being of animals, as well as to improve their management and enhance animal productive performances [4].

The possibility to implement an efficient system for precision monitoring of behaviour in grazing livestock paired with a systematic recording of animal location is still under investigation. The main challenge is to combine the accuracy in data recording (i.e. obtaining fine-scale and behaviourally interpretable data) with the technical (e.g., duration of battery units) and computational (i.e. algorithms for behaviour classification) efficiency.

In the last decade, various research investigated the potential applications of accelerometers and activity sensors alone [5-7] or, less frequently, in combination with GPS telemetry devices [8; 9] for monitoring livestock behaviour, in order to discriminate among activities $[10 ; 11]$ or to estimate energy expenditure [12]. However, studies addressing classification of cattle behaviour using accelerometers integrated in GPS collars are less common; the aim of our research was to test the capability of Discriminant Analysis, applied to data obtained by built-in activity sensors on GPS collars, to discriminate between various activities in grazing cattle.

\section{Materials and methods}

The study was conducted in two farms located in the north-eastern part of Tuscany, Italy (farm 1:

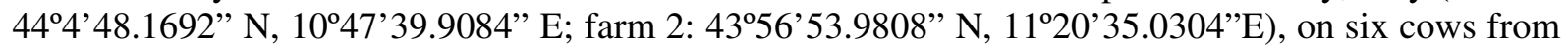
a commercial and a traditional beef breed ( $N=3$ Limousin, $N=3$ Chianina). The animals, separated by breed, were allocated in fenced sown mixed pastures composed on average by $56 \%$ of grassland 
and $44 \%$ of woodland, where they grazed continuously from April to November. Animals in farm 1 ( $N=1$ Limousin, $N=1$ Chianina) rotationally grazed on pastures after the first cut, with an average rotation length of 51 days from April to July and of 10 days from August to November. The extensions of the grazing areas were variable, ranging from 3.3 to 88.3 hectares. In farm 2, animals ( $N=2$ Limousin, $N=2$ Chianina) grazed in a 38 hectares enclosure during the entire study period. All enclosures had accesses to natural (i.e. streams) or artificial watering points. All the observed cows were in good health conditions and multiparous. Two cows (Limousin) were not pregnant during the entire experimental period, while only one animal (a Chianina cow) had calved prior the beginning of the experiment (days in milk at the start of observations: 70). A Chianina gave birth to her calf approximately two months after the beginning of the experiment, while the remaining two cows calved after the end of the observation period.

The animals were equipped with GPS/GSM collars (PRO Light - Vectronic Aerospace GmbH, Berlin; collar weight $\sim 1.7 \mathrm{~kg}$ ) recording their location every hour for the entire pasture season, with the exception of one cow which lost the collar for one week during the month of June. Each collar unit was provided with an activity sensor measuring acceleration on three axes perpendicularly oriented 48 times per second (Fig.1). The device automatically scaled raw acceleration data into levels ranging from 0 to 255 . Every 152 seconds, an average activity value was stored for forward-backward movements ( $X$ axis) and sideways/rotary movements ( $Y$ axis).

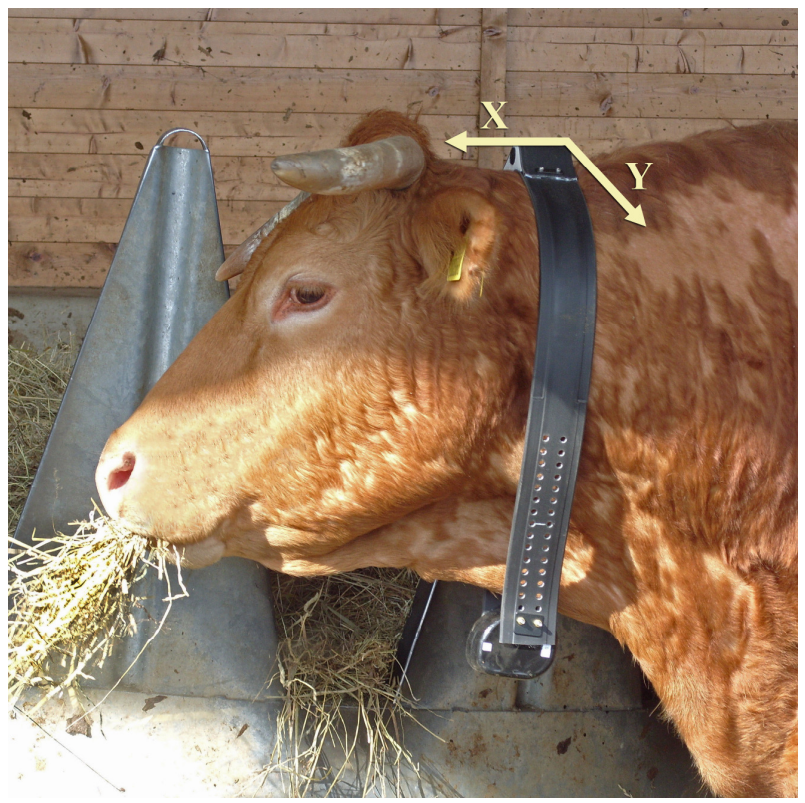

Fig.1. Limousin cow fitted with GPS PRO Light collar: the accelerometer is integrated in the top slot and records horizontal activity along the $X$ (forward-backward movements) and $Y$ (sideways and rotary movements) directions

The calibration of predictive models to discriminate among activities and to infer animal behaviour requires synchronized visual observations of collared individuals. Thus, direct observations in synchrony with collars measurements were conducted during 11 days, for a total amount of 120 hours. In particular, the observations were carried out during two periods of the pasture season: the early-mid, from April to July (hereafter spring/summer), and the later period, from September to November (hereafter autumn). Average minimum temperatures were $5.7^{\circ} \mathrm{C}$ and $2.2^{\circ} \mathrm{C}$, while average maximum temperatures were $30.5^{\circ} \mathrm{C}$ and $24.5^{\circ} \mathrm{C}$, for spring/summer and autumn respectively. Behaviour was measured by scan-sampling at 30 -seconds intervals, and classified in four categories following the ethogram described in McLennan et al. [13]: resting (which includes lying and standing), rumination (both lying and standing), grazing and walking. Location and activity data were downloaded directly from the collar only once, after the end of the monitoring period.

Location data were screened and only accurate positions, obtained using at least 4 satellites and with Dilution of Precision < 10, were retained. Euclidean distances between two consecutive locations (Steplength) were calculated in GIS environment (QGIS 2.18.12). The activity values on $X$ and $Y$ axes 
were highly correlated $\left(r_{\text {pearson }}=0.86\right)$, thus a set of derivate variables was computed basing on their difference (DiffXY), mean (MeanXY) and variance (VarXY). In addition, the logarithm (base 10) of these six predictors was calculated, thus obtaining six further variables $(\log X, \log Y, \log D i f f X Y$, $\log$ MeanXY, $\log$ VarXY, logSteplength). Then, each of the four observed behavioural categories was paired with the corresponding activity values and their derivate variables. The classification of behaviours was implemented with Discriminant Analysis (DA) using Gaussian Finite Mixture Models (R 3.4.1, package "mclust").

Several models were preliminarily tested, using different sets of variables as predictors, and we reported the ones with the higher performances of classification, which were achieved by adopting separate models for the two breeds and, within breed, for two season-related periods (spring/summer and autumn). Walking activity was misclassified in more than $80 \%$ of cases, while rumination and resting behaviour were mutually mistaken. Feeding and walking were thus merged to obtain active behaviours, and rumination and resting were classified as inactive behaviours. In order to validate the classification models, 5 -folds cross-validation was iterated 1,000 times by using a bootstrap procedure. For each run, the confusion matrix obtained from the classification algorithm was extracted in order to evaluate the performances of DA procedure, by computing sensitivity $(S n)$, specificity $(S p)$, precision $(P r)$ and accuracy $(A c)$ as follows:

$$
\begin{gathered}
S n=T P /(T P+F N) \\
S p=T N /(T N+F P) \\
P r=T P /(T P+F P) \\
A c=(T P+T N) /(T P+T N+F P+F N)
\end{gathered}
$$

where $T P$ - true positives;

$T N$ - true negatives;

$F P$ - false positives;

$F N-$ false negatives.

\section{Results and discussion}

The highest rate of correct classification (i.e. sensitivity) of active and inactive behaviours was obtained by adopting separate models by breed and season. It appears thus that season-related environmental effects (e.g., weather conditions, phenology of pasture) as well as the breed-specific habits have to be taken into account when inferring behaviour in grazing animals, since they produce significant alterations in intensity of activity. On average, Chianina cows displayed a more unquiet temperament, which resulted in higher activity levels compared to Limousin cows. The outcome is consistent with the behavioural peculiarities of the two breeds, which acknowledge greater docility in Limousin breed. Indeed, commercial breeds have undergone a more intensive selection involving also behavioural traits.

The set of predictors included in the four final models is reported in Table 1.

Variables included as predictors in the classification models

Table 1

\begin{tabular}{|c|c|c|l|}
\hline Model & Breed & Period & \multicolumn{1}{c|}{ Variables } \\
\hline A & Limousin & Spring/Summer & $X, Y$ \\
\hline B & Limousin & Autumn & $\log X, Y$, DiffXY, MeanXY, logVarXY \\
\hline C & Chianina & Spring/Summer & $X, Y$, DiffXY,MeanXY, logVarXY \\
\hline D & Chianina & Autumn & $\log X, Y$, DiffXY, MeanXY, VarXY, logSteplength \\
\hline
\end{tabular}

The activity values recorded on the $X$ and $Y$ axes, both in the untransformed and in the logarithm form, were included in each model, thus resulting the primary variables in classifying active and inactive behaviours. In the models $\mathrm{B}, \mathrm{C}$ and $\mathrm{D}$, further derivate variables contributed to improve the performances of classification, specifically the difference between activity values recorded on the $X$ 
and $Y$ axes as well as their mean and variance. We observed that during grazing bouts (i.e. grass feeding) the values recorded on the $X$ axis were regularly greater than those recorded on the $Y$ axis, while the opposite occurred during rumination, thus we assumed that the difference between these activity values (DiffXY) would have contributed significantly in the identification of grazing and rumination bouts. In effects, the inclusion of this variable appeared to improve the efficiency of correct assignment of active behaviours after merging the behaviour classes. Grazing indeed is a complex activity encompassing a set of various head, neck and body movements and, particularly when tearing off the grass, neck is engaged in a typical forward-backward motion which is detected by the sensor in the collar. Rumination conversely, despite including some dynamic movements as chewing, regurgitation and swallowing of ingesta [5], produces little changes in acceleration when the sensor is placed around the animal's neck, while the accelerometer is more sensitive to head and neck movement unrelated to this activity. As a result, rumination intervals were frequently misclassified, being assigned mainly to resting behavior or to other activities, since occasional head movements, owed for instance to insect harassment or to grooming activity, determined an increase in recorded activity values, as also observed in similar research [8; 14]. MeanXY and $\operatorname{VarXY}$ variables represent the average overall activity level and an additional measure of the distance between $X$ and $Y$ values, respectively, however they cannot be directly associated to a specific behaviour. Steplength contributed to improve the efficiency of classification only in one occurrence (model D), revealing that broad-scale recording of movement rate cannot be utilized as suitable indicator of the animal motion status (i.e. knowing if the animal is walking or standing) at finer scales. When interested in inferring animal behaviour at the minutes temporal resolution, collars should be scheduled to record positions at a time scale as close as possible to the one of the activity sensor. Anyway, it is clear that such sampling effort would not be compatible with long-term monitoring of cattle behaviour at pasture due to limitations in battery duration.

After bootstrap resampling, the discriminant analysis correctly classified on average over $86 \%$ of intervals in the validation dataset. These overall results are comparable to the ones obtained in similar studies conducted on other species or using different devices, which reported overall accuracies ranging from $77 \%$ to $94 \%[5 ; 7 ; 13 ; 15 ; 16]$.

Table 2

Performances of classification for active and inactive behaviours in Limousin cows

\begin{tabular}{|l|c|c|c|c|}
\hline \multirow{2}{*}{ Limousin } & \multicolumn{2}{c|}{ Spring-Summer } & \multicolumn{2}{c|}{ Autumn } \\
\cline { 2 - 5 } & Active & Inactive & Active & Inactive \\
\hline Sensitivity, \% & $92.0 \pm 4.2$ & $90.6 \pm 5.7$ & $85.6 \pm 6.0$ & $87.6 \pm 4.3$ \\
\hline Specificity, \% & $90.6 \pm 5.7$ & $92.0 \pm 4.2$ & $87.6 \pm 4.3$ & $85.6 \pm 6.0$ \\
\hline Precision, \% & $92.9 \pm 4.3$ & $89.5 \pm 5.3$ & $82.6 \pm 5.3$ & $90.1 \pm 3.9$ \\
\hline Accuracy, \% & \multicolumn{2}{|c|}{$91.4 \pm 3.1$} & \multicolumn{2}{c|}{$86.8 \pm 2.7$} \\
\hline Number of intervals & 200 & 149 & 255 & 376 \\
\hline
\end{tabular}

Table 3

Performances of classification for active and inactive behaviours in Chianina cows

\begin{tabular}{|l|c|c|c|c|}
\hline \multirow{2}{*}{\multicolumn{1}{|c|}{ Chianina }} & \multicolumn{2}{c|}{ Spring-Summer } & \multicolumn{2}{c|}{ Autumn } \\
\cline { 2 - 5 } & Active & Inactive & Active & Inactive \\
\hline Sensitivity, \% & $96.2 \pm 3.8$ & $95.0 \pm 3.4$ & $87.6 \pm 11.4$ & $67.7 \pm 7.8$ \\
\hline Specificity, \% & $95.0 \pm 3.4$ & $96.2 \pm 3.8$ & $67.7 \pm 7.8$ & $87.6 \pm 11.4$ \\
\hline Precision, \% & $85.7 \pm 8.5$ & $98.8 \pm 1.1$ & $46.0 \pm 8.1$ & $94.9 \pm 4.3$ \\
\hline Accuracy, \% & \multicolumn{2}{|c|}{$95.2 \pm 2.6$} & \multicolumn{2}{c|}{$72.4 \pm 5.2$} \\
\hline Number of intervals & 124 & 412 & 93 & 300 \\
\hline
\end{tabular}

In general, the use of accelerometers returning processed values (e.g., scaled or averaged over a time interval) or positioned on the animal's neck results in a reduced detectability of specific behaviours and in lower performances of correct classification. For instance, the possibility to identify 
rumination requires devices placed in correspondence of the animal's jaw [5; 7; 16]. Despite these limitations, the overall results of this experiment equalized the classification potential of sensors returning raw acceleration data and improved the outcomes of experimentations conducted with comparable devices.

The performances in the assignment of the two behavioural categories differed among breeds and between season-related periods (Table 2, Table 3). Both in Limousin and in Chianina cows, the best performances of classification were attained during the warm season (i.e. spring/summer), both evaluating the overall accuracy of the model and considering sensitivity within each behaviour. Consistenly, during the cold season, the activity levels of the animals recorded by the sensor were reduced compared to spring/summer and values associated with active behaviour were more similar to those associated with inactive behaviour. A divergent outcome emerged when considering seasonality: in the warm season, the accuracy of classification was higher for the Chianina dataset while during the autumn period, the classification model applied to Limousin dataset displayed a largely higher accuracy. This result could be again explained as a consequence of breed specific behaviour in relation to environmental conditions. In Figure 2 are provided, as an example, the classified data points for the validation dataset of model A, including misclassified and correctly assigned behaviours. Discriminant analysis assigned correctly active intervals more frequently than the inactive ones with the exception of model B, however in general the model tended to overestimate active behaviours, especially during the cold season.

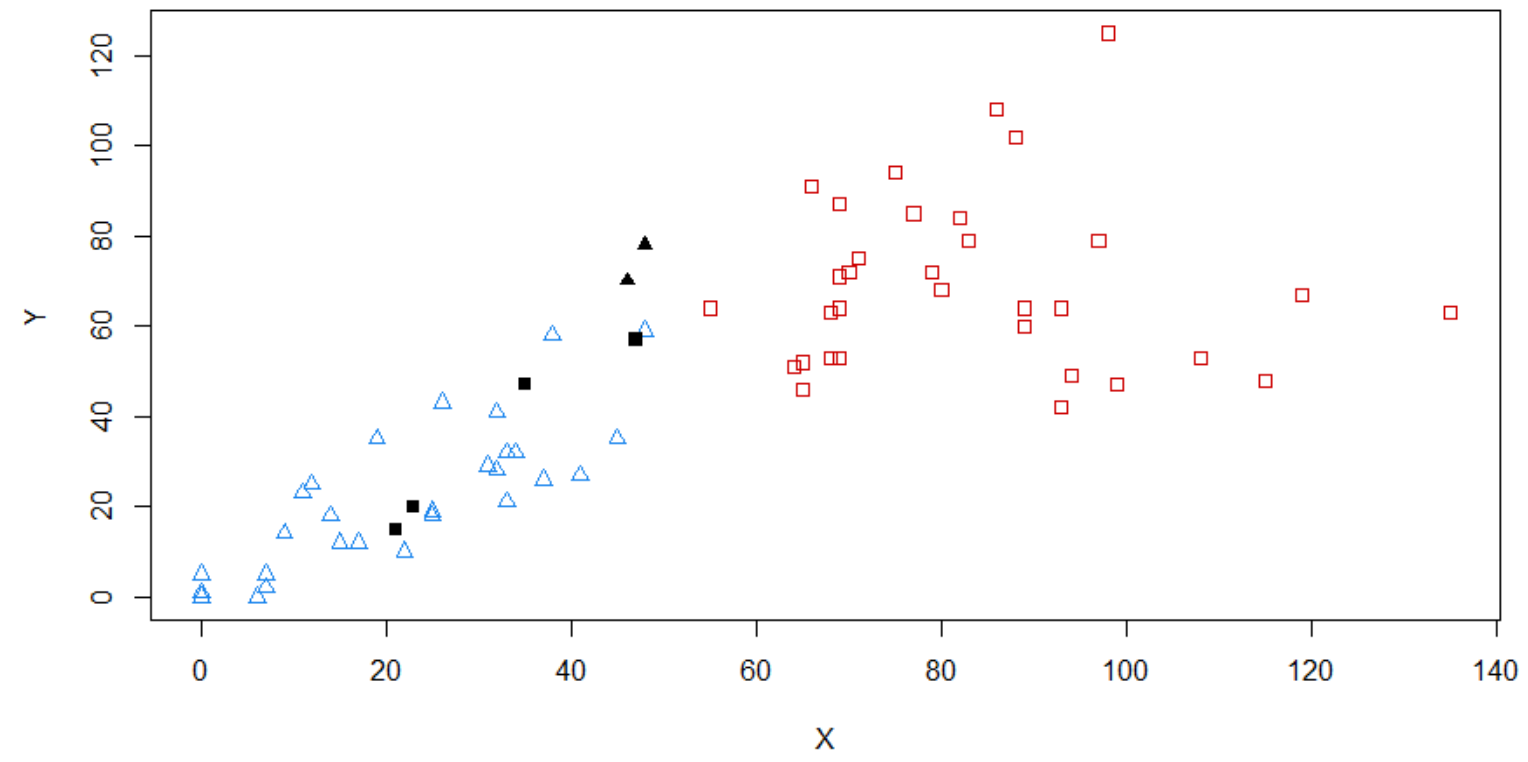

Fig.2. Classification performed on validation dataset for model A: correct classifications for inactive (blue triangles) and active (red squares) behaviours and classification errors (black solid triangles and squares)

In our experiment classification precision, expressing the proportion of observed intervals within each behavioural class to those classified, was reasonably high for inactive behaviours (on average over $93 \%$ ) and comparable to the results obtained in similar studies [5; 16]. Precision attained an adequate level also for active behaviours (82.6-92.9\%), with the exception of the Chianina dataset in the autumn season, when the ability of the model to classify correctly active behaviours was the lowest.

\section{Conclusions}

GPS collars combined with activity sensors represent an effective technique to track movements of grazing livestock and, at the same time, to infer animal behaviour. The use of averaged and scaled activity data along two directions (forward-backward and sideways movements) over at a time epoch of 152 seconds proved to be useful in discriminating between active and inactive behaviours with reasonable accuracy. 
However, the identification of specific behaviours as rumination or travelling would require data to be collected with different modalities. Since the activity sensor is placed on the animals' neck, the recorded data are likely to be affected by occasional head movements. Thus, by recording raw acceleration data on three axes and by scheduling a fine-scale position acquisition rate, the performances of classification would be largely improved both in terms of percentage of correct classification and as a number of identifiable behavioural classes.

We observed that specific behavioural expressions might require the adoption of separate classification models, as in the case of diverse breeds. Moreover, as opposed to comparable studies that implemented direct observations along few consecutive days, our approach allowed to encompass seasonal variations of the activity state of the animals. Similar changes, related to their biology or to the effect of environmental conditions, may thus require the adoption of separate predictive models in order to improve the efficiency of classification.

\section{Acknowledgements}

The authors thank the farms "Il Butale" and "Fattoria di Valdastra" who provided valuable contribution to this research.

This research was part of a PRIN (2008), funded by MIUR, Italy.

\section{References}

[1] Ponzetta M. P., Cervasio F., Crocetti C., Messeri A., Argenti G. Habitat improvements with wildlife purposes in a grazed area on the Apennine Mountains. Italian Journal of Agronomy, 5(3), 2010, pp. 233-238.

[2] Dumont B., Rook A. J., Coran C., Röver K. U. Effects of livestock breed and grazing intensity on biodiversity and production in grazing systems. 2. Diet selection. Grass and Forage Science, 62(2), 2007, pp.159-171.

[3] Owen-Smith N., Fryxell J. M., Merrill E. H. Foraging theory upscaled: the behavioural ecology of herbivore movement. Philosophical Transactions of the Royal Society B: Biological Sciences, 365(1550), 2010, pp.2267-2278.

[4] Berckmans D. Precision livestock farming technologies for welfare management in intensive livestock systems. Scientific and Technical Review of the Office International des Epizooties, 33(1), 2014, pp.189-196.

[5] Watanabe N., Sakanoue S., Kawamura K., Kozakai T. Development of an automatic classification system for eating, ruminating and resting behavior of cattle using an accelerometer. Grassland Science, 54(4), 2008, pp.231-237.

[6] Diosdado J. A. V., Barker Z. E., Hodges H. R., Amory J. R., Croft D. P., Bell N. J., Codling E. A. Classification of behaviour in housed dairy cows using an accelerometer-based activity monitoring system. Animal Biotelemetry, 3(1):15, 2015.

[7] Alvarenga F.A.P., Borges I., Palkovič L., Rodina J., Oddy V.H., Dobos R.C. Using a three-axis accelerometer to identify and classify sheep behaviour at pasture. Applied Animal Behaviour Science, 181, 2016, pp.91-99.

[8] Ungar E. D., Henkin Z., Gutman M., Dolev A., Genizi A., Ganskopp D. Inference of animal activity from GPS collar data on free-ranging cattle. Rangeland Ecology \& Management, 58(3), 2005, pp.256-266.

[9] González L. A., Bishop-Hurley G. J., Handcock R. N., Crossman C. Behavioral classification of data from collars containing motion sensors in grazing cattle. Computers and Electronics in Agriculture, 110, 2015, pp. 91-102.

[10] Scheibe K. M., Gromann C. Application testing of a new three-dimensional acceleration measuring system with wireless data transfer (WAS) for behavior analysis. Behavior Research Methods, 38(3), 2006, pp.427-433.

[11] Martiskainen P., Järvinen M., Skön J. P., Tiirikainen J., Kolehmainen M., Mononen J. Cow behaviour pattern recognition using a three-dimensional accelerometer and support vector machines. Applied Animal Behaviour Science, 119(1), 2009, pp.32-38.

[12] Miwa M., Oishi K., Nakagawa Y., Maeno H., Anzai H., Kumagai H., Okano K., Tobioka H., Hirooka $\mathrm{H}$. Application of overall dynamic body acceleration as a proxy for estimating the energy 
expenditure of grazing farm animals: relationship with heart rate. PloS one, 10(6), e0128042, 2015.

[13] McLennan K. M., Skillings E. A., Rebelo C. J., Corke M. J., Moreira M. A. P., Morton A. J., Constantino-Casas F. Validation of an automatic recording system to assess behavioural activity level in sheep (Ovis aries). Small Ruminant Research, 127, 2015, pp.92-96.

[14]Pépin D., Renaud P. C., Decuq F. Identifying activity patterns from activity counters in ETHOSYS ${ }^{\circledR}$ collars on red deer. Applied Animal Behaviour Science, 96(1), 2006, pp.103-114.

[15] Moreau M., Siebert S., Buerkert A., Schlecht E. Use of a triaxial accelerometer for automated recording and classification of goats' grazing behaviour. Applied Animal Behaviour Science, 119 (3), 2009, pp.158-170.

[16] Giovanetti V., Decandia M., Molle G., Acciaro M., Mameli M., Cabiddu A., Cossu R., Serra M. G., Manca C., Rassu S. P. G., Dimauro C. Automatic classification system for grazing, ruminating and resting behaviour of dairy sheep using a tri-axial accelerometer. Livestock Science, 196, 2017, pp.42-48. 